\title{
Distributed Cognition: Interactions Between Individuals and Artifacts
}

Eric Wilson, Colleen Seifert, Steven J Durning, Dario Torre, Michelle Daniel

In distributed cognition, bidirectional interactions between individuals and artifacts (i.e., medical charts, computers, imaging technology) facilitate medical decision making. Because cognition is dispersed among team members and their work tools, the system has greater capacity for developing complex diagnostic and treatment plans. Additionally, the distributed nature of cognitive processes allows information to be accessed and modified by multiple individuals, teams, and information systems across space and time. However, access to certain elements of the distributed cognitive system can also be limited by an individual's horizon of observation; that is, their ability to directly interact with specific providers and patients, access to clinical notes and records, and testing data such as imaging.

Example: A 25-year-old male is brought to a local hospital after a motor vehicle collision (MVC) in which he was the unrestrained driver. The paramedics report significant vehicle damage and a prolonged extraction. He is complaining of severe chest and abdominal pain and has parasternal, abdominal and thoracic spine tenderness on initial exam. The emergency physician (EP) obtains a pan-CT. The radiologist notes multiple rib fractures and an aortic dissection. The EP arranges air-medical transfer to a trauma center. The chart, but not the original images, are sent with the patient. On arrival at the trauma center, the patient is tachycardic and hypotensive. The receiving EP discusses the case with the trauma surgeon, and the patient is rushed to an operating room (OR). Post-operatively, the patient is paraplegic and a T7 burst fracture is found.

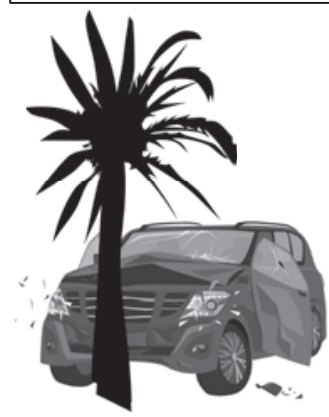

Patient tells paramedics that he has severe pain in his chest and abdomen

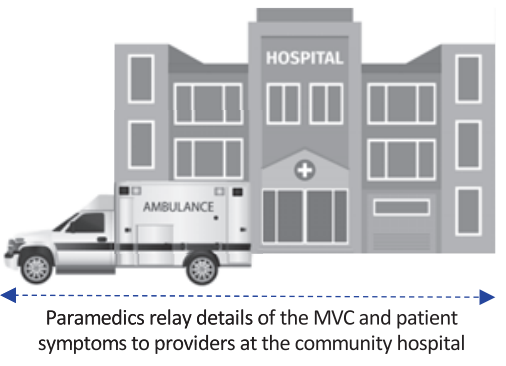

EP at community hospital elicits parasternal, abdominal, and

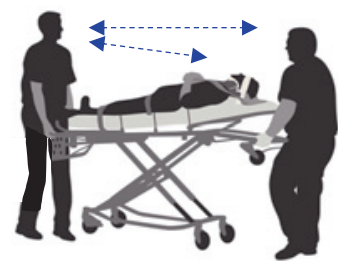

thoracic spine tenderness on exam
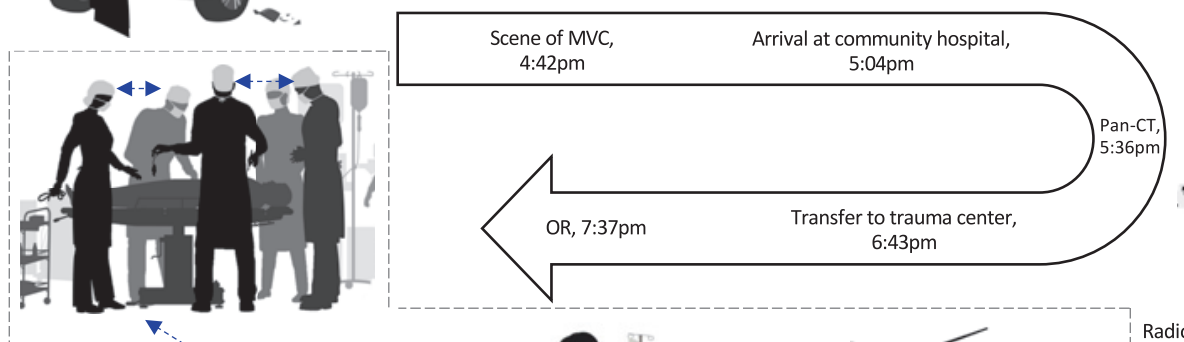
Paramedics relay details of the MVC and patient

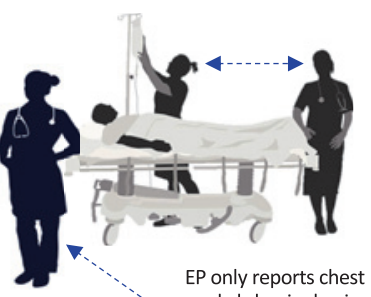

EP only reports chest
and abdominal pain and abdominal
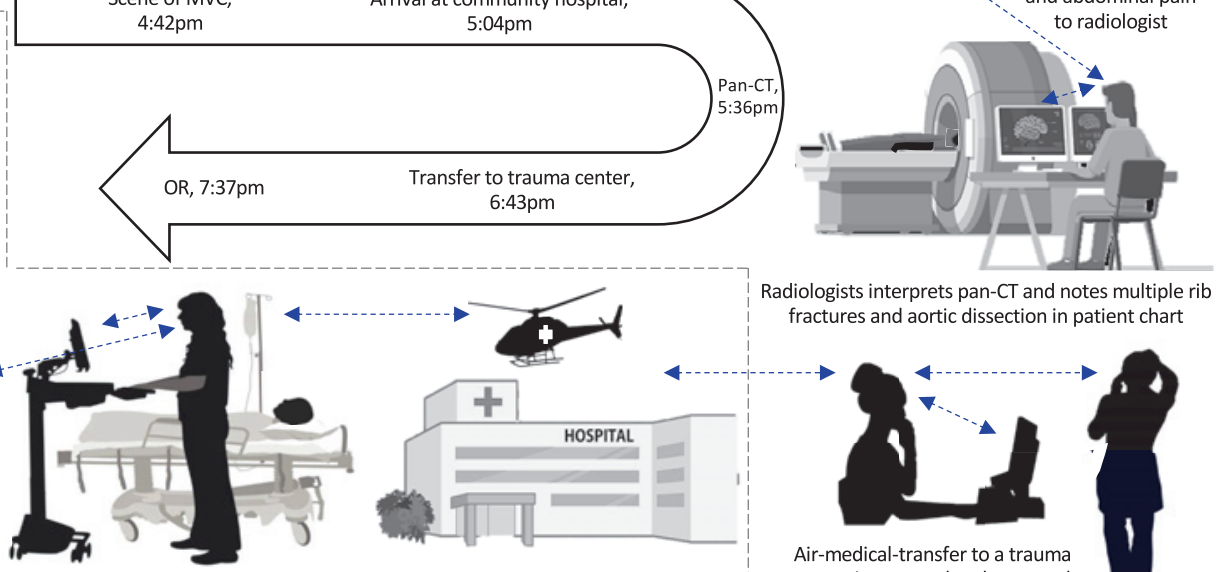

Receiving EP and trauma surgeon discuss the details of the case and decide to operate immediately

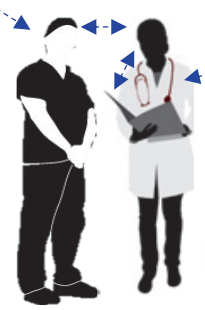

Patient is noted to be tachycardic and hypotensive on arrival

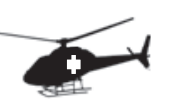

Radiologists interprets pan-CT and notes multiple rib fractures and aortic dissection in patient chart

\begin{tabular}{|c|c|c|}
\hline Interactions between people and artifacts or tools & Horizon of observation at trauma center & $\begin{array}{c}\text { } \\
\text { Information processing sequence across space and time }\end{array}$ \\
\hline
\end{tabular}

In this example, a distributed cognitive network (DCN) is created as individual healthcare workers (i.e., paramedics, EPs, radiologist, trauma surgeon) gather historical (i.e., chest and abdominal pain), physical (i.e., parasternal, abdominal, and thoracic spine tenderness), and imaging (i.e., pan-CT) data that is exchanged via team interactions and/or externally represented through artifacts (i.e. patient chart, $\mathrm{CT}$ images). Individual providers can expand the DCN by documenting their impressions (i.e., radiologist dictates multiple rib fractures and traumatic aortic dissection in patient's chart) or verbalizing their thoughts with others (i.e., EP reports chest and abdominal pain, but fails to mention thoracic spine tenderness to the radiologist). Ultimately, details from the scene of the MVC provided by the paramedics, signs and symptoms noted by the EP at the community hospital, and the radiologist's interpretation of the pan-CT are communicated to the receiving EP and surgeon at the trauma center. The boundaries of the DCN span space and time, enhancing the ability of providers at the trauma center to act. However, because the CT images were not delivered to the trauma center, the providers develop a treatment plan based on the radiologist's note in the chart. Their limited horizon of observation (i.e., inability to view CT images) causes them to miss the T7 burst fracture until they note his paraplegia post-operatively.

References: 1) Hutchins, E. (1995). Cognition in the wild. Cambridge, MA: The MIT Press.

Disclaimer: The views expressed herein are those of the authors and not necessarily those of the Department of Defense or other federal agencies. 
*Corresponding author: Michelle Daniel, Office of Medical Student Education, University of Michigan Medical School, 6123 Taubman Health Sciences Library, 1135 Catherine, Ann Arbor, 48109-0624, MI, USA, E-mail: micdan@umich.edu

Eric Wilson: Medical Student, University of Michigan Medical School, Ann Arbor, MI, USA

Colleen Seifert: Department of Psychology, University of Michigan College of Literature Science and the Arts, Ann Arbor, MI, USA
Steven J. Durning and Dario Torre: Internal Medicine, Uniformed Services University of the Health Sciences, Bethesda, MD, USA

https://doi.org/10.1515/dx-2020-0012

Published online June 19, 2020

Supplementary Material: The licenses for the images are provided in the online version of this article (https://doi.org/10.1515/dx-2020-0012). 